\title{
Afatinib alone and in combination with vinorelbine or paclitaxel, in patients with HER2-positive breast cancer who failed or progressed on prior trastuzumab and/or lapatinib (LUX-Breast 2): an open-label, multicenter, phase II trial
}

\author{
Tamas Hickish $^{1}$ - Ajay Mehta ${ }^{2} \cdot$ Mei-Ching Liu ${ }^{3}$. Chiun-Sheng Huang ${ }^{4} \cdot$ Rajendra Singh Arora $^{5}$. \\ Yuan-Ching Chang ${ }^{6} \cdot$ Youngsen Yang $^{7,8} \cdot$ Vladimir Vladimirov $^{9} \cdot$ Minish Jain $^{10} \cdot$ Janice Tsang $^{11} \cdot$ Karine Pemberton $^{12}$. \\ Behbood Sadrolhefazi ${ }^{13} \cdot$ Xidong Jin $^{14} \cdot$ Ling-Ming Tseng $^{15}$
}

Accepted: 11 September 2021 / Published online: 9 February 2022

(C) The Author(s) 2022

\begin{abstract}
Purpose Resistance to HER2 (ErbB2)-targeted therapy may be mediated by other members of the ErbB family. We investigated the efficacy and safety of the irreversible ErbB family blocker, afatinib, alone as first-line therapy in the advanced setting and in combination with vinorelbine or paclitaxel for those who progressed on afatinib monotherapy, in female patients with metastatic breast cancer who had failed or progressed on prior HER2-targeted therapy in the early disease setting.

Methods In this phase II, single-arm, two-part study (ClinicalTrials.gov: NCT01271725), patients in part A received afatinib $40 \mathrm{mg} /$ day in 21-day cycles until disease progression or intolerable adverse events (AEs). Patients with progressive disease could then receive afatinib plus weekly vinorelbine $25 \mathrm{mg} / \mathrm{m}^{2}$ or paclitaxel $80 \mathrm{mg} / \mathrm{m}^{2}$ until disease progression or intolerable AEs (part B). The primary endpoint was confirmed objective response rate (RECIST v1.1).

Results Eighty-seven patients were enrolled and 74 were treated in part A (median age: 51 years [range 27-76]; 31 [42\%] estrogen receptor-positive, 26 [35\%] progesterone receptor-positive). Of these, 39 (53\%) patients went on to receive afatinib plus vinorelbine (13 patients) or paclitaxel (26 patients) in part B. Thirteen (18\%) and $12(31 \%)$ patients achieved an objective response in parts $\mathrm{A}$ and $\mathrm{B}$, respectively. The most common treatment-related AEs with afatinib monotherapy (any/ grade $\geq 3)$ were diarrhea $(68 \% / 8 \%)$ and rash $(49 \% / 4 \%)$. Combination therapy was generally well tolerated, with no additive toxicity observed.

Conclusion Afatinib treatment, alone or in combination with vinorelbine or paclitaxel, was associated with objective responses in $\geq 18 \%$ of patients with metastatic breast cancer for whom prior HER2-targeted therapy has failed. Treatmentrelated AEs were generally manageable, with few grade $\geq 3$ AEs reported.
\end{abstract}

Trial registration ClinicalTrials.gov, NCT01271725, registered 1 July 2011.

Keywords Afatinib $\cdot$ ErbB $\cdot$ HER2 $\cdot$ Metastatic breast cancer $\cdot$ Resistance

\section{Introduction}

The human epidermal growth factor receptor 2 (HER2/ Neu/ErbB2) is amplified and/or overexpressed in approximately $15 \%$ of human breast cancers [1] and plays a direct

Dr. Liu has sadly passed away since the preparation of this article.

Tamas Hickish

tamas.hickish@rbch.nhs.uk

Extended author information available on the last page of the article role in tumor development $[2,3]$. In patients with breast cancer, HER2 amplification/overexpression is a significant predictor of shorter overall survival and time to relapse [4, 5]. Targeted [6-8] treatment against HER2 with the monoclonal antibodies trastuzumab and pertuzumab, the small molecule tyrosine kinase inhibitors lapatinib, neratinib, and tucatinib, and the antibody-chemotherapy conjugates trastuzumab emtansine and trastuzumab deruxtecan has markedly improved outcomes in patients with HER2-positive breast cancer [9-13]. These treatments are now approved and recommended for use in this setting $[14,15]$. 
Despite the availability of anti-HER 2 therapies, primary and acquired resistance to these treatments frequently occur and represent a significant clinical challenge [16-18]. Novel agents to treat patients with HER2-positive breast cancer who have exhausted all current options are therefore urgently needed. Diverse mechanisms of resistance to anti-HER2 therapy, mediated by aberrations in numerous alternative pathways, have been identified, including those involving other receptors in the ErbB family, such as the epidermal growth factor receptor ([EGFR]/ErbB1), HER3 (ErbB3), and HER4 (ErbB4) [16, 17, 19, 20]. Some evidence suggests that targeting multiple members of the ErbB family improves outcomes compared with targeting one receptor alone [12, 21], although not all studies support these findings [22].

Afatinib, an irreversible ErbB family blocker, is a specific, potent inhibitor of HER2, EGFR, and HER4, which blocks signaling from all homo- and heterodimers formed by ErbB family members [23]. We hypothesized that broad inhibition of multiple ErbB family members may help to overcome resistance to prior HER2-targeted therapy and findings from previous phase I and II trials of afatinib in HER2-positive metastatic breast cancer were encouraging [24-27]. In one phase II study, four of 35 evaluable patients (11\%) experienced a partial response to afatinib monotherapy after progression on trastuzumab and 15 patients (43\%) had stable disease [25]. In the neoadjuvant setting, singleagent afatinib demonstrated clinical activity comparable to that of trastuzumab and lapatinib in HER2-positive patients with locally advanced breast cancer [27].

The present trial aimed to investigate the use of afatinib, both alone and in combination with chemotherapy, in a larger population of patients with HER2-positive metastatic breast cancer who had failed previous HER2-targeted therapy. Previous studies of afatinib and vinorelbine in patients with breast cancer suggested that these two agents could be safely combined, with potential clinical benefit $[24,26]$. In keeping with current use of trastuzumab [28], afatinib was to be continued beyond the point of disease progression.

\section{Material and methods}

\section{Patients and study design}

In this open-label, phase II, single-arm, two-part study, patients were enrolled at 27 sites across Asia and Europe (Russia, the United Kingdom, Poland, Hong Kong, Taiwan, and India). At two sites, including the site in Poland, patients were enrolled but not treated. In part A, patients received afatinib monotherapy; in part $\mathrm{B}$, patients with disease progression on monotherapy during part $\mathrm{A}$ were eligible to receive combination therapy with afatinib and either vinorelbine or paclitaxel.
The study enrolled female patients aged 18 years or over with histologically confirmed HER2-overexpressing metastatic breast cancer. Patients must have failed or progressed on prior trastuzumab or lapatinib or trastuzumab and lapatinib combination therapy, in the neoadjuvant and/or adjuvant setting. Other inclusion criteria included at least one measurable lesion according to the Response Evaluation Criteria in Solid Tumors version 1.1 (RECIST v1.1) [29], an Eastern Cooperative Oncology Group (ECOG) performance status of $0-2$, life expectancy of at least 6 months, and an archived tissue sample available for central re-assessment of HER2 status. Patients eligible for part B must also have progressed on afatinib monotherapy in the first-line metastatic setting (part A) and be eligible for treatment with vinorelbine or paclitaxel.

Key exclusion criteria were as follows: presence of active brain metastases, prior first-line therapy for metastatic breast cancer; radiotherapy (except short-course palliative radiotherapy to non-target lesions), chemotherapy, immunotherapy, any investigational drug, trastuzumab or lapatinib treatment, or surgery (other than biopsy) within 4 weeks prior to trial treatment; hormone therapy for breast cancer within 2 weeks prior to trial treatment; or prior EGFR/ HER2-targeted treatment other than trastuzumab or lapatinib in the neoadjuvant and/or adjuvant setting (parts A and B) or afatinib in the first-line setting (part B).

All patients provided written informed consent. The trial was carried out in accordance with the Declaration of Helsinki and the protocol was approved by the independent ethics committees and/or institutional review boards of the participating centers. The study is registered with ClinicalTrials.gov (NCT01271725).

\section{Study treatment}

In part A, afatinib was administered orally at a dose of $40 \mathrm{mg} /$ day until disease progression or intolerable adverse events (AEs). Once disease progression occurred, patients received weekly intravenous infusions of either vinorelbine $25 \mathrm{mg} / \mathrm{m}^{2}$ or paclitaxel $80 \mathrm{mg} / \mathrm{m}^{2}$ in addition to daily afatinib until disease progression or intolerable AEs. The choice of chemotherapeutic agent was made by the investigator, providing patients were eligible for either treatment. Treatment cycles were 21 days.

For the first instance of disease progression in part A patients could receive short-course palliative radiotherapy if necessary, while continuing afatinib monotherapy. Patients could enter part B 3-4 weeks after completion of radiotherapy.

In the event of any grade $\geq 3$ treatment-related $\mathrm{AE}$, grade 2 diarrhea persisting for $\geq 2$ consecutive days despite adequate anti-diarrheal medication/hydration, grade $\geq 2$ nausea and/or vomiting persisting for $\geq 7$ consecutive days despite 
antiemetic treatment/hydration, or grade $\geq 2$ worsening of renal function, afatinib treatment was paused until the $\mathrm{AE}$ had recovered to grade $\leq 1$ or baseline. Treatment was then resumed at a 10-mg lower dose, with a minimum dose of $20 \mathrm{mg}$ (treatment was discontinued if the patient was already receiving a dose of $20 \mathrm{mg}$ ).

\section{Endpoints}

The primary endpoint was confirmed objective response, defined as complete response (CR) plus partial response (PR). Secondary endpoints were as follows: best overall response (objective response with and without confirmation) during each treatment period; duration of objective response (without confirmation), defined as the time from first objective response to time of progression, death, or censoring; progression-free survival (PFS), defined for three time intervals: from the start of monotherapy to the time of first disease progression/death, from the start of combination therapy to time of second disease progression/death, and from the start of monotherapy to the time of second disease progression/death; and safety.

\section{Assessments}

Efficacy was evaluated according to RECIST v1.1; response was evaluated by the investigator. Tumor assessment by computed tomography (CT) scan was performed at baseline, every 6 weeks (two courses) after the start of treatment, and before the commencement of combination therapy in part B. Tumor assessment was not required prior to the start of combination therapy if a CT scan detecting progressive disease on afatinib monotherapy was conducted within 3 weeks prior to the commencement of combination treatment. However, if radiotherapy had been given prior to commencing combination therapy, a tumor assessment had to be performed to document the baseline status of combination therapy. Disease progression was determined based solely on clinical assessment or on both clinical judgment and tumor imaging. AEs and laboratory parameters were graded according to the Common Terminology Criteria for Adverse Events Version 3.0.

\section{Statistical analysis}

Based on a binomial probability distribution and an assumed underlying objective response rate (ORR) of $10 \%$, a total sample size of 80 patients would be expected to give an $82 \%$ probability of observing at least six responders. All analyses were descriptive and exploratory. PFS was assessed based on the Kaplan-Meier method for each part separately, and point estimates together with confidence intervals (CIs; based on Greenwood's method) were calculated for median PFS.

\section{Results}

\section{Patients}

Between July 4, 2011 and October 16, 2013, 87 patients were enrolled, of whom 74 were treated in part A (Fig. 1). Of the 74 patients treated in part A, 39 (53\%) went on to receive combination treatment in part $\mathrm{B} ; 13$ patients received afatinib and vinorelbine and 26 patients received afatinib and paclitaxel. While the study was ongoing, the data monitoring committee of a related trial, LUX-Breast 1 , recommended termination of the afatinib and vinorelbine combination treatment arm in that study due to insufficient clinical benefit and higher rates of discontinuations, dose reductions, serious AEs, and deaths, relative to the comparator arm, trastuzumab, and vinorelbine [30]. As a precautionary measure, no further patients were enrolled into the afatinib and vinorelbine arm of this study from May 3, 2013. Later on, further enrollment into part A was also stopped. Patients who were receiving and benefitting from afatinib plus vinorelbine before May 3, 2013 were permitted to continue. Ongoing treatment in part $\mathrm{A}$ and treatment with afatinib plus paclitaxel in part B was not affected.

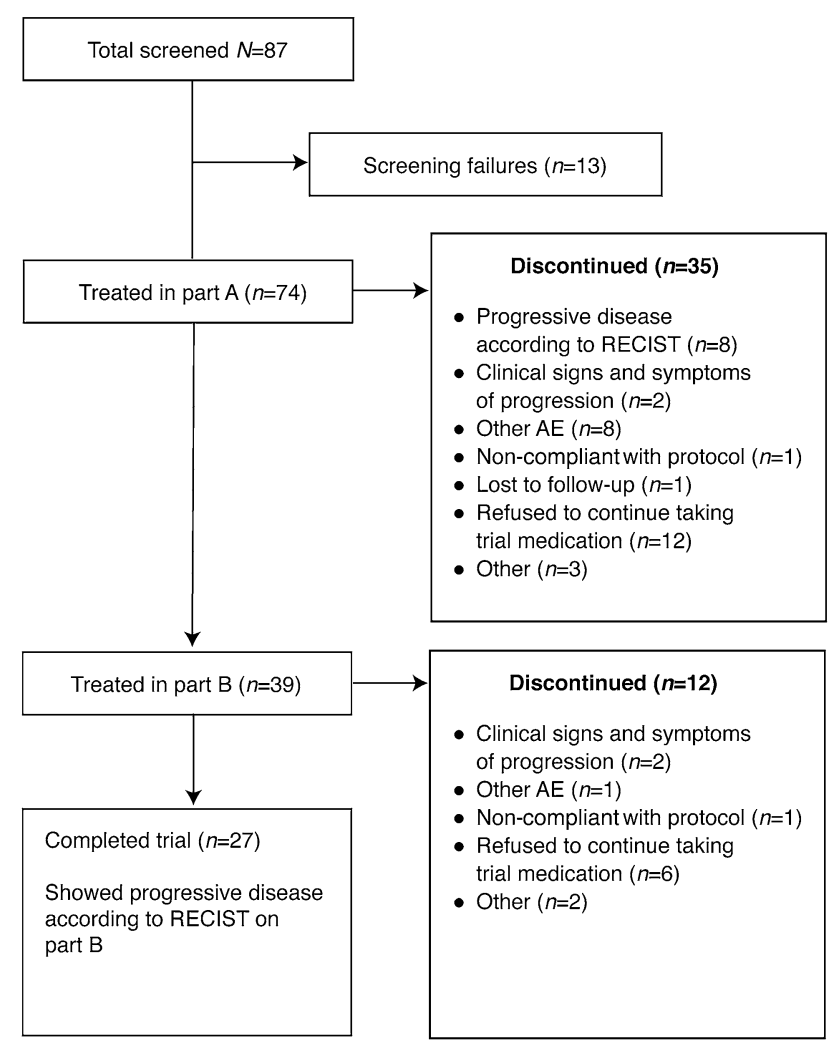

Fig. 1 Patient disposition. AE adverse event, RECIST Response Evaluation Criteria in Solid Tumors 
Table 1 Baseline characteristics

\begin{tabular}{|c|c|c|}
\hline Characteristic & Afatinib (part A; $N=74$ ) & $\begin{array}{l}\text { Afatinib + vinorelbine or } \\
\text { paclitaxel (part } \mathrm{B} ; N=39)\end{array}$ \\
\hline Median age, years (range) & $51(27-76)$ & $52(27-73)$ \\
\hline \multicolumn{3}{|l|}{ Race, $n(\%)$} \\
\hline Asian & $47(64)$ & $22(56)$ \\
\hline Indian & $16(22)$ & $5(13)$ \\
\hline Taiwanese or Chinese & $31(42)$ & $17(44)$ \\
\hline White & $27(36)$ & $17(44)$ \\
\hline \multicolumn{3}{|l|}{ Smoking status, $n(\%)$} \\
\hline Never smoked & $69(93)$ & $37(95)$ \\
\hline Ex-smoker & $2(3)$ & $1(3)$ \\
\hline Current smoker & $3(4)$ & $1(3)$ \\
\hline \multicolumn{3}{|l|}{ Alcohol status, $n(\%)$} \\
\hline Non-drinker & $63(85)$ & $32(82)$ \\
\hline Mean BMI, $\mathrm{kg} / \mathrm{m}^{2}(\mathrm{SD})$ & $25.8(4.5)$ & $27.1(4.8)$ \\
\hline \multicolumn{3}{|l|}{ ECOG PS, $n(\%)$} \\
\hline 0 & $46(62)$ & $21(54)$ \\
\hline 1 & $27(36)$ & $18(46)$ \\
\hline 2 & $1(1)$ & 0 \\
\hline Menopausal status, $n(\%)$ & & $\mathrm{NE}$ \\
\hline Premenopausal & $16(22)$ & \\
\hline Perimenopausal & $3(4)$ & \\
\hline Postmenopausal & $55(74)$ & \\
\hline Median time from first diagnosis, years (range) & $2.4(0.6-8.8)$ & NE \\
\hline Estrogen receptor status at first diagnosis, $n(\%)$ & & $\mathrm{NE}$ \\
\hline Positive & $31(42)$ & \\
\hline Negative & $43(58)$ & \\
\hline Progesterone receptor status at first diagnosis ${ }^{\mathrm{a}}, n(\%)$ & & $\mathrm{NE}$ \\
\hline Positive & $26(35)$ & \\
\hline Negative & $47(64)$ & \\
\hline HER2 status at first diagnosis, $n(\%)$ & & NE \\
\hline Positive & $72(97)$ & \\
\hline Negative & $2(3)$ & \\
\hline Previous HER2-targeted therapy & & NE \\
\hline Trastuzumab & $64(86)$ & \\
\hline Lapatinib & $6(8)$ & \\
\hline Trastuzumab and lapatinib & $4(5)$ & \\
\hline Metastatic sites at baseline, $n(\%)$ & & $\mathrm{NE}$ \\
\hline 1 & $23(31)$ & \\
\hline 2 & $24(32)$ & \\
\hline 3 & $17(23)$ & \\
\hline$\geq 4$ & $10(14)$ & \\
\hline Location of metastases, $n(\%)$ & & $\mathrm{NE}$ \\
\hline Lung & $43(58)$ & \\
\hline Liver & $32(43)$ & \\
\hline Skin & $7(9)$ & \\
\hline Pleura & $3(4)$ & \\
\hline Bone & $14(19)$ & \\
\hline Lymph nodes & $42(57)$ & \\
\hline Contralateral breast cancer & $8(11)$ & \\
\hline Brain & $1(1)$ & \\
\hline Other & $8(11)$ & \\
\hline
\end{tabular}

$B M I$ body mass index, ECOG PS Eastern Cooperative Oncology Group performance status, $N E$ not evaluated, $S D$ standard deviation

${ }^{a}$ Data missing for one patient 
Baseline characteristics for patients in part A and part B are shown in Table 1. In total, $63(85 \%)$ patients had infiltrating ductal carcinoma, six (8\%) patients had infiltrating lobular carcinoma, one (1\%) patient had tubular carcinoma, one (1\%) patient had inflammatory breast cancer, and five (7\%) patients had other tumor histology (multiple tumor types were reported for some patients).

Over the whole study (part A and part B combined), median exposure to afatinib was 166.0 days (range 1-1562 days). Median exposure to study medication was 83.5 days (range 1-1491 days) during part A, 92.0 days (range 29-266 days) in patients who received afatinib and vinorelbine in part $B$, and 128.5 days (range 1-1205 days) in patients who received afatinib and paclitaxel in part $\mathrm{B}$.

\section{Efficacy}

Thirteen (18\%) patients had a confirmed objective response to afatinib monotherapy (part A), including one (1\%) patient with a confirmed CR and 12 patients (16\%) with a confirmed PR. Thirty-three (45\%) patients had stable disease (Table 2). The patient with a confirmed CR was a 48 -year-old Asian patient with moderately differentiated infiltrating ductal carcinoma. She had received prior treatment with lapatinib and tamoxifen and six courses of cyclophosphamide, doxorubicin, and 5-fluorouracil. CR was sustained for 211 days. Further two patients had an unconfirmed PR. Median duration of objective response (regardless of confirmation) was 168.5 days.

In part B, $12(31 \%)$ patients had a confirmed PR during combined therapy with afatinib and vinorelbine or paclitaxel and a further five patients had an unconfirmed PR. Eighteen (46\%) patients had stable disease. Median duration of objective response regardless of confirmation was 125.0 days.

Table 2 Confirmed ORR

\begin{tabular}{lrc}
\hline Patients, $n(\%)$ & $\begin{array}{l}\text { Afatinib } \\
\text { (part A; } \\
N=74)\end{array}$ & $\begin{array}{l}\text { Afatinib + vinorelbine or } \\
\text { paclitaxel (part B; } N=39)\end{array}$ \\
\hline $\begin{array}{l}\text { Disease control } \\
(\mathrm{CR}+\text { PR + SD) }\end{array}$ & $46(62)$ & $30(77)$ \\
Objective response & $13(18)$ & $12(31)$ \\
$\quad$ CR & $1(1)$ & 0 \\
PR & $12(16)$ & $12(31)$ \\
SD & $33(45)$ & $18(46)$ \\
$\quad$ Unconfirmed CR/PR & $2(3)$ & $5(13)$ \\
PD & $21(28)$ & $4(10)$ \\
Not evaluable & $7(9)$ & $5(13)$ \\
\hline
\end{tabular}

$C R$ complete response, $O R R$ objective response rate, $P D$ progressive disease, $P R$ partial response, $S D$ stable disease

${ }^{a} \mathrm{CR} / \mathrm{PR} \geq 35$ days from first study drug administration in part $\mathrm{A}$ but not confirmed $\geq 28$ days later
Median PFS was 86 days (95\% CI 72-127) in part A (Fig. 2a), 135 days (95\% CI 95-224) in part B (Fig. 2b), and 267 days (95\% CI 207-360) when considering the entire study (Fig. 2c).

\section{Safety}

In total, 64 (86\%) patients experienced treatment-related AEs in part A (Table 3). In part B, rates of treatment-related AEs were higher in patients receiving combination therapy with vinorelbine (92\%) than with paclitaxel (85\%). In total, $30(41 \%)$ patients experienced an AE necessitating a reduction in the dose of afatinib during part $\mathrm{A}$; fewer patients required dose reductions during part $\mathrm{B}$ (afatinib plus vinorelbine: $15 \%$; afatinib plus paclitaxel: $27 \%$; Table 3 ).

The most common treatment-related AEs in patients receiving afatinib monotherapy in part A were (any/grade $3 / 4)$ diarrhea $(68 \% / 8 \%)$ and rash $(49 \% / 8 \%)$. In patients receiving afatinib with vinorelbine in part $\mathrm{B}$, the most common treatment-related AE of any grade and of grade 3/4 was neutropenia $(62 \% / 38 \%)$. Among patients receiving afatinib with paclitaxel, the most common any grade treatmentrelated AEs were anemia (42\%), alopecia (38\%), and neutropenia (31\%) and the most common grade 3/4 treatmentrelated AE was neutropenia (19\%) (Table 4).

Four patients experienced treatment-related serious adverse events (SAEs) in part A: diarrhea (two patients), herpes zoster, and interstitial lung disease (one patient each). Among the patients receiving afatinib and vinorelbine in part B, two patients had a total of three treatmentrelated SAEs: abdominal pain, febrile neutropenia, and pyrexia. Two patients receiving afatinib and paclitaxel experienced a total of four treatment-related SAEs: azotemia, blood creatinine increased, diarrhea, and nausea.

Across parts A and B, 12 patients experienced fatal AEs, none of which were considered to be related to study treatment by the investigators. All fatal AEs represented disease progression and/or its complications.

\section{Discussion}

When this study was conceived, options for patients with HER2-positive metastatic breast cancer who had failed treatment with taxanes and targeted agents were limited. We therefore investigated whether afatinib, given alone and in combination with vinorelbine or paclitaxel, could be a further treatment option for these patients. Despite the encouraging clinical activity seen in prior studies, enrollment was stopped after 74 patients had entered the study, following the discontinuation of a similar trial, LUX-Breast 1 [30]. This phase III study was terminated after a benefit-risk assessment indicated a low likelihood of improved PFS and lower 
a

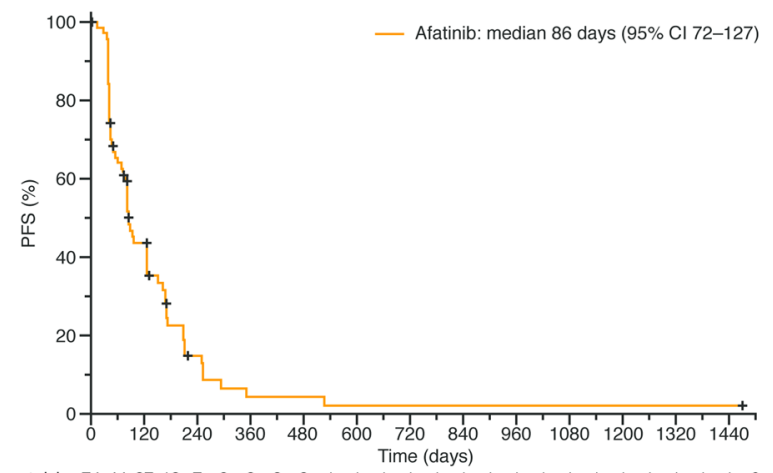

Number at risk $\begin{array}{lllllllllllllllllllllllllll}44 & 44 & 27 & 12 & 7 & 3 & 2 & 2 & 2 & 1 & 1 & 1 & 1 & 1 & 1 & 1 & 1 & 1 & 1 & 1 & 1 & 1 & 1 & 1 & 1 & 0\end{array}$

b
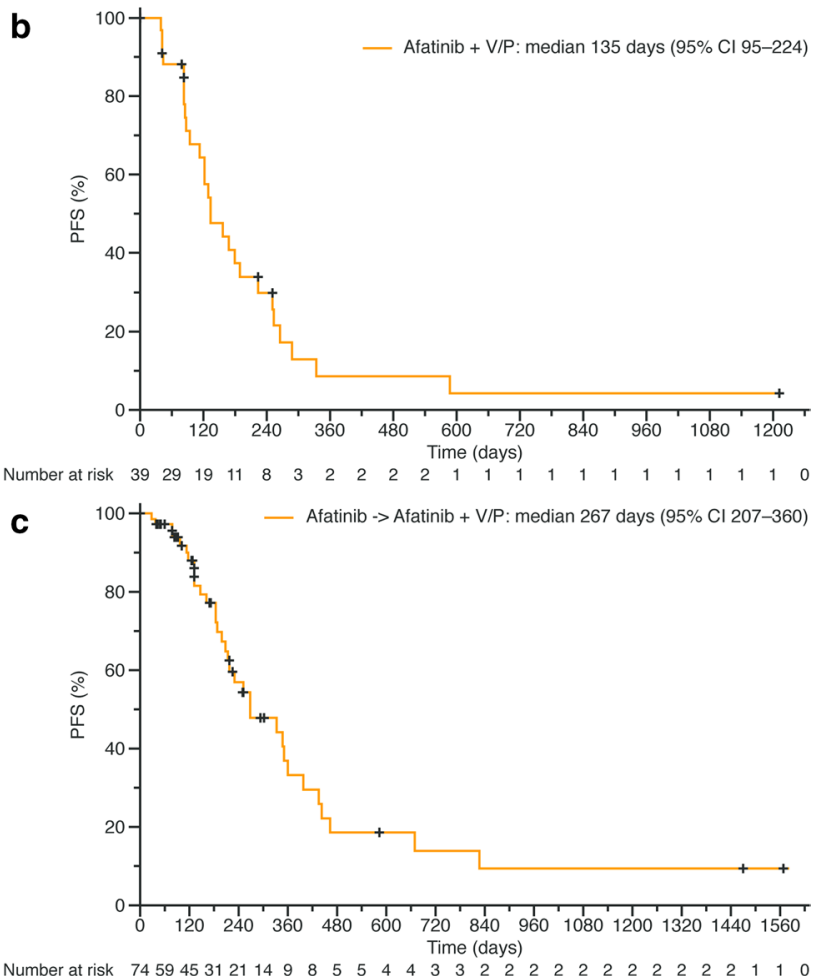

Fig. 2 Progression-free survival during a part A (from the start of afatinib monotherapy to the time of the first disease progression or death); $\mathbf{b}$ part B (from the start of combined treatment with afatinib and vinorelbine or paclitaxel to the time of second disease progression or death); c the entire study (from the start of afatinib monotherapy to the time of second disease progression or death). Afatinib $+V / P$ afatinib combination therapy with either vinorelbine or paclitaxel, $C I$ confidence interval

tolerability, with afatinib and vinorelbine versus trastuzumab and vinorelbine. Similarly, in the phase II LUX-Breast 3 study, which enrolled patients with HER2-positive metastatic breast cancer with progressive brain metastases after trastuzumab, lapatinib, or both, no benefit and higher toxicity were seen with afatinib \pm vinorelbine compared with investigators' choice of treatment [31].

Despite the negative results seen in previous trials, findings from the present study suggest that afatinib monotherapy may be of clinical benefit to some patients with metastatic HER2-positive breast cancer who have failed prior HER2-targeted treatment. Overall, $62 \%$ of patients on afatinib monotherapy had a period of disease control, including one CR that was sustained for 211 days. Further, our data suggest that the combination of afatinib with vinorelbine or paclitaxel was also of clinical benefit to some patients who had progressed on afatinib monotherapy, with disease control achieved in $77 \%$ of patients.

The tolerability of afatinib and vinorelbine was generally comparable to that seen with afatinib and paclitaxel, with similar proportions of patients experiencing treatmentrelated grade $\geq 3$ AEs in both treatment arms. Indeed, the incidence of treatment-related grade $\geq 3$ AEs was only slightly higher in the afatinib combination arms than in the afatinib monotherapy group and the incidence of grade 1-2 events was lower AEs which were consistent with previous studies [25, 32-34] and no apparent adverse interactions between afatinib and vinorelbine or afatinib and paclitaxel were observed.

With phase III studies showing the clinical benefits of the newer anti-HER2 agents, pertuzumab, and trastuzumab emtansine [12, 13], the future role of afatinib for the treatment of HER2-positive patients who have previously been treated with trastuzumab appears to be limited. However, afatinib has shown some activity in other populations of patients with metastatic breast cancer, including patients with triple-negative breast cancer who had progressed following three or fewer lines of chemotherapy [35] and in combination with letrozole in patients with estrogen receptor-positive metastatic breast cancer who had progressed on letrozole monotherapy [36]. Further investigation into the use of afatinib in these settings may therefore be warranted; however, no further development of afatinib for HER2-positive breast cancer is currently planned. The findings from this study show that broad ErbB blockade may be of benefit in overcoming resistance to prior HER2-targeted therapy in only a small number of patients and suggest that, for most patients, novel agents targeting different oncogenic proteins and pathways are needed. Furthermore, now that we have entered the era of precision medicine and personalized medicine, there is still an ongoing urgent need for further specific markers for us to select the most optimal anti-HER2 therapy for this group of patients, as reflected by the achievement of a durable $\mathrm{CR}$ in a patient treated with single-agent afatinib in part A.

\section{Conclusion}

Treatment with afatinib alone provided an objective response of $18 \%$ in patients with metastatic breast cancer who had previously failed or progressed on HER2-targeted therapy 
Table 3 Summary of adverse events in part A (afatinib) and part B (afatinib and vinorelbine or paclitaxel)

\begin{tabular}{|c|c|c|c|}
\hline \multirow[t]{2}{*}{ Patients, $n(\%)$} & \multirow{2}{*}{$\begin{array}{l}\text { Part A } \\
\text { Afatinib }(N=74)\end{array}$} & \multicolumn{2}{|l|}{ Part B } \\
\hline & & $\begin{array}{l}\text { Afatinib + vinorelbine } \\
(N=13)\end{array}$ & $\begin{array}{l}\text { Afatinib + pacli- } \\
\text { taxel }(N=26)\end{array}$ \\
\hline Any AE & $71(96)$ & $13(100)$ & $25(96)$ \\
\hline Treatment-related AE & $64(86)$ & $12(92)$ & $22(85)$ \\
\hline AE leading to dose reduction & $30(41)^{\mathrm{a}}$ & $2(15)^{\mathrm{b}}$ & $7(27)^{\mathrm{c}}$ \\
\hline AE leading to discontinuation & $13(18)^{\mathrm{d}}$ & $2(15)^{\mathrm{e}}$ & $8(31)^{\mathrm{f}}$ \\
\hline Serious AE & $18(24)$ & $5(38)$ & $10(38)$ \\
\hline Grade $\geq 3 \mathrm{AE}$ & $32(43)$ & $8(62)$ & $17(65)$ \\
\hline
\end{tabular}

$A E$ adverse event

${ }^{a}$ Most common: diarrhea (17 [23\%]) and mucosal inflammation (3 [4\%])

${ }^{\mathrm{b}}$ Diarrhea and neutropenia

${ }^{\mathrm{c}}$ Most common: diarrhea (4 [15\%]) and neuropathy peripheral (2 [8\%])

${ }^{\mathrm{d}}$ Most common: diarrhea and neoplasm progression (2 [3\%] each)

${ }^{\mathrm{e}}$ Two patients experienced a total of three AEs: malignant neoplasm progression, neutropenia, and leukopenia

${ }^{\mathrm{f}}$ Most common: asthenia, leukopenia, and vomiting (2 [8\%] each)

Table 4 Most common treatment-related AEs

\begin{tabular}{|c|c|c|c|c|c|c|c|c|c|}
\hline \multirow[t]{3}{*}{ Number of patients, $n(\%)$} & \multirow{2}{*}{\multicolumn{3}{|c|}{$\frac{\text { Part A }}{\text { Afatinib }(N=74)}$}} & \multicolumn{6}{|l|}{ Part B } \\
\hline & & & & \multicolumn{3}{|c|}{ Afatinib + vinorelbine $(N=13)$} & \multicolumn{3}{|c|}{ Afatinib + paclitaxel $(N=26)$} \\
\hline & Grade $1-2$ & Grade 3 & Grade 4 & Grade $1-2$ & Grade 3 & Grade 4 & Grade $1-2$ & Grade 3 & Grade 4 \\
\hline Treatment-related AEs & $44(59)$ & $20(27)$ & 0 & $6(46)$ & $4(31)$ & $2(15)$ & $12(46)$ & $9(35)$ & $1(4)$ \\
\hline Diarrhea & $44(59)$ & $6(8)$ & 0 & $2(15)$ & $1(8)$ & 0 & $8(31)$ & $2(8)$ & 0 \\
\hline Neutropenia & $1(1)$ & 0 & 0 & $3(23)$ & $3(23)$ & $2(15)$ & $3(12)$ & $5(19)$ & 0 \\
\hline Rash & $33(45)$ & $3(4)$ & 0 & $2(15)$ & 0 & 0 & $3(12)$ & 0 & 0 \\
\hline Anemia & $3(4)$ & 0 & 0 & $1(8)$ & 0 & 0 & $10(38)$ & $1(4)$ & 0 \\
\hline Alopecia & $2(3)$ & 0 & 0 & $1(8)$ & 0 & 0 & $10(38)$ & 0 & 0 \\
\hline Fatigue & $4(5)$ & 0 & 0 & 0 & 0 & 0 & $5(19)$ & 0 & 0 \\
\hline Asthenia & $3(4)$ & 0 & 0 & $2(15)$ & 0 & 0 & $5(19)$ & 0 & 0 \\
\hline Leukopenia & 0 & 0 & 0 & $3(23)$ & 0 & 0 & $5(19)$ & 0 & 0 \\
\hline Mucosal inflammation & $13(18)$ & $1(1)$ & 0 & 0 & 0 & 0 & $1(4)$ & 0 & 0 \\
\hline $\begin{array}{l}\text { Palmar-plantar erythrodysesthe- } \\
\text { sia syndrome }\end{array}$ & $10(14)$ & $2(3)$ & 0 & $1(8)$ & 0 & 0 & $2(8)$ & $1(4)$ & 0 \\
\hline Paronychia & $9(12)$ & 0 & 0 & $2(15)$ & 0 & 0 & $1(4)$ & 0 & 0 \\
\hline Peripheral sensory neuropathy & 0 & 0 & 0 & 0 & 0 & 0 & $4(15)$ & 0 & 0 \\
\hline Mouth ulceration & $7(9)$ & 0 & 0 & $2(15)$ & 0 & 0 & $2(8)$ & 0 & 0 \\
\hline Nausea & $5(7)$ & 0 & 0 & 0 & 0 & 0 & $3(12)$ & $1(4)$ & 0 \\
\hline Dermatitis & $3(4)$ & $1(1)$ & 0 & $2(15)$ & 0 & 0 & $1(4)$ & 0 & 0 \\
\hline Neuropathy peripheral & 0 & 0 & 0 & 0 & 0 & 0 & $3(12)$ & 0 & 0 \\
\hline Vomiting & $3(4)$ & 0 & 0 & 0 & 0 & 0 & $2(8)$ & 0 & $1(4)$ \\
\hline AST increased & $3(4)$ & 0 & 0 & 0 & 0 & 0 & $2(8)$ & 0 & 0 \\
\hline ALT increased & $2(3)$ & 0 & 0 & 0 & 0 & 0 & $2(8)$ & $1(4)$ & 0 \\
\hline Erythema multiforme & 0 & $2(3)$ & 0 & 0 & 0 & 0 & 0 & 0 & 0 \\
\hline
\end{tabular}

AEs are presented by preferred term. No patient experienced a grade 5 treatment-related AE. Includes events reported for at least $10 \%$ of patients (grades 1-2) in any treatment group or any grade 3 or 4 event that was reported in more than one patient. Additional grade 3 AEs affecting one patient each in the afatinib monotherapy group were herpes zoster, interstitial lung disease, dermatitis acneiform, and skin ulcer. Additional grade 3 AEs affecting one patient each in the afatinib and paclitaxel group were skin fissures, skin ulcer, weight decreased, blood creatinine increased, and white blood cell count decreased. An additional grade $3 \mathrm{AE}$ affecting one patient in the afatinib and vinorelbine group was pyrexia

$A E s$ adverse events, $A L T$ alanine aminotransferase, $A S T$ aspartate aminotransferase 
and objective response increased to $31 \%$ in patients whose disease progressed on afatinib monotherapy and were treated with afatinib in combination with vinorelbine or paclitaxel. These findings indicate that afatinib may provide some clinical benefit in this patient group. The AEs observed were in line with the known safety profile of afatinib. However, enrollment for this study was stopped early, following discontinuation of LUX-Breast 1 [30], for which a benefit-risk assessment favored trastuzumab in combination with vinorelbine over afatinib in combination with vinorelbine. No new trials for afatinib in HER2-positive breast cancer are planned; however, as afatinib has shown activity in other populations of patients with metastatic breast cancer [35, 36], further investigation in different specific indications together with companion biomarkers may be warranted.

Acknowledgements We thank the patients, their families, the investigators, and the staff who participated in the trial. Medical writing assistance, supported financially by Boehringer Ingelheim Pharmaceuticals, Inc, was provided by Christina Jennings and Jim Sinclair of GeoMed, an Ashfield company, part of UDG Healthcare plc, during the preparation of this manuscript.

Author contributions $\mathrm{TH}$ was the principal investigator of the trial. TH and JT contributed to study conception and design. TH, JT, KP, Y-CC, L-MT, VV, MJ, C-SH, YY, M-CL, AM, and RSA were responsible for collection and assembly of data. TH, JT, XJ, C-SH, and BS were responsible for data analysis and interpretation. TH, JT, XJ, KP, Y-CC, L-MT, VV, MJ, C-SH, YY, M-CL, AM, RSA, and BS were responsible for drafting and/or writing the manuscript. All authors provided final approval of the manuscript and agreed to be accountable for all aspects of the work, including ensuring questions related to the accuracy or integrity of any part of the work are appropriately investigated and resolved

Funding This study was sponsored/funded by Boehringer Ingelheim. The study sponsor was involved in study design, collection, and interpretation of data, writing of the report, and the decision to submit the article for publication.

Data availability To ensure independent interpretation of clinical study results, Boehringer Ingelheim grants all external authors access to relevant material, including participant-level clinical study data, as needed by them to fulfill their role and obligations as authors under the ICMJE criteria. Clinical study documents and participant clinical study data are available to be shared on request after publication of the primary manuscript in a peer-reviewed journal, and if regulatory activities are complete and other criteria met as per the BI Policy on Transparency and Publication of Clinical Study Data (https://www.mystudywin dow.com/msw/datasharing). Bona fide, qualified scientific and medical researchers are eligible to request access to the clinical study data with corresponding documentation describing the structure and content of the datasets. Upon approval, and governed by a Legal Agreement, data are shared in a secured data-access system for a limited period of 1 year, which may be extended upon request. Prior to providing access, clinical study documents and data will be examined, and, if necessary, redacted and de-identified, to protect the personal data of study participants and personnel, and to respect the boundaries of the informed consent of the study participants. Researchers should use the https://vivli.org/ link to request access to study data and visit https:// www.mystudywindow.com/msw/datasharing for further information.
Code availability Not applicable.

\section{Declarations}

Conflict of interest M-CL has received travel and accommodation costs and expenses from Pfizer. C-SH has been involved with advisory councils or committees for Roche, Amgen, Eli Lilly, and Pfizer, received honoraria from Roche, Amgen, Eli Lilly, Pfizer, and Novartis, and received grants or funds from AstraZeneca, Daiichi Sankyo, EirGenix, Eli Lilly, MSD, Novartis, OBI Pharma, Pfizer, and Roche. JT has received honoraria from Amgen, NanoString, Novartis, Pfizer, and Roche and has received consulting fees from NanoString and Pfizer. KP, BS and XJ are current employees of Boehringer Ingelheim. TH, AM, RSA, Y-CC, YY, VV, MJ, and L-MT declare no potential conflict of interest.

Ethical approval Ethical approval was received by the Southampton and West Hampshire Research Ethics Committee (REC). The clinical trial protocol, patient information leaflet, informed consent form, and other locally required documents were received by the Independent Ethics Committees (IECs) and/or Institutional Review Boards (IRBs) of the participating centers prior to study initiation. These IECs and/or IRBs met the requirements of the International Council on Harmonization (ICH) Harmonized Tripartite Guideline for Good Clinical Practice (GCP) and local legislation. The competent authority (Medicines and Healthcare Regulatory Agency, UK) approved the trial on 25th November 2010.

Informed consent Not applicable.

Open Access This article is licensed under a Creative Commons Attribution 4.0 International License, which permits use, sharing, adaptation, distribution and reproduction in any medium or format, as long as you give appropriate credit to the original author(s) and the source, provide a link to the Creative Commons licence, and indicate if changes were made. The images or other third party material in this article are included in the article's Creative Commons licence, unless indicated otherwise in a credit line to the material. If material is not included in the article's Creative Commons licence and your intended use is not permitted by statutory regulation or exceeds the permitted use, you will need to obtain permission directly from the copyright holder. To view a copy of this licence, visit http://creativecommons.org/licenses/by/4.0/.

\section{References}

1. Noone AM, Cronin KA, Altekruse SF et al (2017) Cancer incidence and survival trends by subtype using data from the Surveillance Epidemiology and End Results program, 1992-2013. Cancer Epidemiol Biomarkers Prev 26(4):632-641. https://doi. org/10.1158/1055-9965.EPI-16-0520

2. Di Fiore PP, Pierce JH, Kraus MH, Segatto O, King CR, Aaronson SA (1987) erbB-2 is a potent oncogene when overexpressed in NIH/3T3 cells. Science 237(4811):178-182

3. Guy CT, Webster MA, Schaller M, Parsons TJ, Cardiff RD, Muller WJ (1992) Expression of the neu protooncogene in the mammary epithelium of transgenic mice induces metastatic disease. Proc Natl Acad Sci USA 89(22):10578-10582. https://doi.org/10.1073/ pnas.89.22.10578

4. Slamon DJ, Clark GM, Wong SG, Levin WJ, Ullrich A, McGuire WL (1987) Human breast cancer: correlation of relapse and survival with amplification of the HER-2/neu oncogene. Science 235(4785): 177-182 
5. Wright C, Angus B, Nicholson S et al (1989) Expression of c-erbB-2 oncoprotein: a prognostic indicator in human breast cancer. Cancer Res 49(8):2087-2090

6. Miles J, White Y (2018) Neratinib for the treatment of early-stage HER2-positive breast cancer. J Adv Pract Oncol 9(7):750-754

7. Murthy RK, Loi S, Okines A et al (2020) Tucatinib, trastuzumab, and capecitabine for HER2-positive metastatic breast cancer. N Engl J Med 382(6):586. https://doi.org/10.1056/NEJMx190039. Erratum for Murthy RK, Loi S, Okines A et al (2020) Tucatinib, trastuzumab, and capecitabine for HER2-positive metastatic breast cancer. N Engl J Med 382(7):597-609

8. Modi S, Saura C, Yamashita T et al (2019) Trastuzumab deruxtecan in previously treated HER2-positive breast cancer. N Engl J Med 382(7):610-621. https://doi.org/10.1056/NEJMoa1914510

9. Geyer CE, Forster J, Lindquist D et al (2006) Lapatinib plus capecitabine for HER2-positive advanced breast cancer. N Engl J Med 355(26):2733-2743. https://doi.org/10.1056/NEJMoa0643 20

10. Krop IE, Kim SB, Martin AG et al (2017) Trastuzumab emtansine versus treatment of physician's choice in patients with previously treated HER2-positive metastatic breast cancer (TH3RESA): final overall survival results from a randomised open-label phase 3 trial. Lancet Oncol 18(6):743-754. https://doi.org/10.1016/S14702045(17)30313-3

11. Slamon DJ, Leyland-Jones B, Shak S et al (2001) Use of chemotherapy plus a monoclonal antibody against HER2 for metastatic breast cancer that overexpresses HER2. N Engl J Med 344(11):783-792. https://doi.org/10.1056/NEJM20010315344 1101

12. Swain SM, Baselga J, Kim SB et al (2015) Pertuzumab, trastuzumab, and docetaxel in HER2-positive metastatic breast cancer. N Engl J Med 372(8):724-734. https://doi.org/10.1056/NEJMo a1413513

13. Verma S, Miles D, Gianni L et al (2012) Trastuzumab emtansine for HER2-positive advanced breast cancer. N Engl J Med 367(19):1783-1791. https://doi.org/10.1056/NEJMoa1209124

14. Cardoso F, Senkus E, Costa A et al (2018) 4th ESO-ESMO International Consensus Guidelines for advanced breast cancer (ABC 4). Ann Oncol 29(8):1634-1657. https://doi.org/10.1093/ annonc/mdy 192

15. Giordano SH, Temin S, Chandarlapaty S et al (2018) Systemic therapy for patients with advanced human epidermal growth factor receptor 2-positive breast cancer: ASCO Clinical Practice Guideline Update. J Clin Oncol 36(26):2736-2740. https://doi. org/10.1200/JCO.2018.79.2697

16. Canfield K, Li J, Wilkins OM et al (2015) Receptor tyrosine kinase ERBB4 mediates acquired resistance to ERBB2 inhibitors in breast cancer cells. Cell Cycle 14(4):648-655. https:// doi.org/10.4161/15384101.2014.994966

17. Luque-Cabal M, Garcia-Teijido P, Fernandez-Perez Y, SanchezLorenzo L, Palacio-Vazquez I (2016) Mechanisms behind the resistance to trastuzumab in HER2-amplified breast cancer and strategies to overcome it. Clin Med Insights Oncol 10(Suppl 1):21-30. https://doi.org/10.4137/CMO.S34537

18. Ruprecht B, Zaal EA, Zecha J et al (2017) Lapatinib resistance in breast cancer cells is accompanied by phosphorylationmediated reprogramming of glycolysis. Cancer Res 77(8):18421853. https://doi.org/10.1158/0008-5472.CAN-16-2976

19. Garrett JT, Olivares MG, Rinehart C et al (2011) Transcriptional and posttranslational up-regulation of HER3 (ErbB3) compensates for inhibition of the HER2 tyrosine kinase. Proc Natl Acad Sci USA 108(12):5021-5026. https://doi.org/10.1073/pnas. 1016140108

20. Ritter CA, Perez-Torres M, Rinehart C et al (2007) Human breast cancer cells selected for resistance to trastuzumab in vivo overexpress epidermal growth factor receptor and ErbB ligands and remain dependent on the ErbB receptor network. Clin Cancer Res 13(16):4909-4919. https://doi.org/10.1158/1078-0432. CCR-07-0701

21. Blackwell KL, Burstein HJ, Storniolo AM et al (2010) Randomized study of lapatinib alone or in combination with trastuzumab in women with ErbB2-positive, trastuzumab-refractory metastatic breast cancer. J Clin Oncol 28(7):1124-1130. https:// doi.org/10.1200/JCO.2008.21.4437

22. Piccart-Gebhart M, Holmes E, Baselga J et al (2016) Adjuvant lapatinib and trastuzumab for early human epidermal growth factor receptor 2-positive breast cancer: results from the randomized phase III adjuvant lapatinib and/or trastuzumab treatment optimization trial. J Clin Oncol 34(10):1034-1042. https:// doi.org/10.1200/JCO.2015.62.1797

23. Solca F, Dahl G, Zoephel A et al (2012) Target binding properties and cellular activity of afatinib (BIBW 2992), an irreversible ErbB family blocker. J Pharmacol Exp Ther 343(2):342350. https://doi.org/10.1124/jpet.112.197756

24. Goh G, Schmid R, Guiver K et al (2016) Clonal evolutionary analysis during HER2 blockade in HER2-positive inflammatory breast cancer: a phase II open-label clinical trial of afatinib +/- vinorelbine. PLoS Med 13(12):e1002136. https://doi.org/ 10.1371/journal.pmed.1002136

25. Lin NU, Winer EP, Wheatley D et al (2012) A phase II study of afatinib (BIBW 2992), an irreversible ErbB family blocker, in patients with HER2-positive metastatic breast cancer progressing after trastuzumab. Breast Cancer Res Treat 133(3):10571065. https://doi.org/10.1007/s10549-012-2003-y

26. Mukai H, Masuda N, Ishiguro H et al (2015) Phase I trial of afatinib plus vinorelbine in Japanese patients with advanced solid tumors, including breast cancer. Cancer Chemother Pharmacol 76(4):739-750. https://doi.org/10.1007/ s00280-015-2826-4

27. Rimawi MF, Aleixo SB, Rozas AA et al (2015) A neoadjuvant, randomized, open-label phase II trial of afatinib versus trastuzumab versus lapatinib in patients with locally advanced HER2positive breast cancer. Clin Breast Cancer 15(2):101-109. https:// doi.org/10.1016/j.clbc.2014.11.004

28. Wong HH, Collins J, McAdam K, Wilson C (2014) Trastuzumab beyond progression in advanced breast cancer: national guidance versus oncologist's decision. Oncology 86(1):22-23. https://doi. org/10.1159/000356876

29. Eisenhauer EA, Therasse P, Bogaerts J et al (2009) New response evaluation criteria in solid tumours: revised RECIST guideline (version 1.1). Eur J Cancer 45(2):228-247. https://doi.org/10. 1016/j.ejca.2008.10.026

30. Harbeck N, Huang CS, Hurvitz S et al (2016) Afatinib plus vinorelbine versus trastuzumab plus vinorelbine in patients with HER2-overexpressing metastatic breast cancer who had progressed on one previous trastuzumab treatment (LUX-Breast 1): an open-label, randomised, phase 3 trial. Lancet Oncol 17(3):357366. https://doi.org/10.1016/S1470-2045(15)00540-9

31. Cortes J, Dieras V, Ro J et al (2015) Afatinib alone or afatinib plus vinorelbine versus investigator's choice of treatment for HER2positive breast cancer with progressive brain metastases after trastuzumab, lapatinib, or both (LUX-Breast 3): a randomised, openlabel, multicentre, phase 2 trial. Lancet Oncol 16(16):1700-1710. https://doi.org/10.1016/S1470-2045(15)00373-3

32. Fumoleau P, Delgado FM, Delozier T et al (1993) Phase II trial of weekly intravenous vinorelbine in first-line advanced breast cancer chemotherapy. J Clin Oncol 11(7):1245-1252. https://doi. org/10.1200/JCO.1993.11.7.1245

33. Perez EA (1998) Paclitaxel in breast cancer. Oncologist 3(6):373-389

34. Seidman AD, Hudis CA, Raptis G, Baselga J, Fennelly D, Norton L (1997) Paclitaxel for breast cancer: the Memorial 
Sloan-Kettering Cancer Center experience. Oncology (Williston Park) 11(3 Suppl 2):20-28

35. Schuler M, Awada A, Harter P et al (2012) A phase II trial to assess efficacy and safety of afatinib in extensively pretreated patients with HER2-negative metastatic breast cancer. Breast Cancer Res Treat 134(3):1149-1159. https://doi.org/10.1007/ s10549-012-2126-1

36. Gunzer K, Joly F, Ferrero JM et al (2016) A phase II study of afatinib, an irreversible ErbB family blocker, added to letrozole in patients with estrogen receptor-positive hormone-refractory metastatic breast cancer progressing on letrozole. Springerplus 5:45. https://doi.org/10.1186/s40064-015-1601-7

Publisher's Note Springer Nature remains neutral with regard to jurisdictional claims in published maps and institutional affiliations.

\section{Authors and Affiliations}

\section{Tamas Hickish $^{1}$ - Ajay Mehta ${ }^{2} \cdot$ Mei-Ching Liu ${ }^{3} \cdot$ Chiun-Sheng Huang ${ }^{4} \cdot$ Rajendra Singh Arora ${ }^{5}$. Yuan-Ching Chang ${ }^{6} \cdot$ Youngsen Yang $^{7,8} \cdot$ Vladimir Vladimirov $^{9} \cdot$ Minish Jain $^{10} \cdot$ Janice Tsang $^{11} \cdot$ Karine Pemberton $^{12}$. Behbood Sadrolhefazi ${ }^{13} \cdot$ Xidong Jin $^{14} \cdot$ Ling-Ming Tseng $^{15}$}

1 Royal Bournemouth Hospital/Bournemouth University, Castle Ln E, Bournemouth BH7 7DW, UK

2 Central India Cancer Research Institute, Nagpur, India

3 Koo Foundation Sun Yat-Sen Cancer Center, Taipei, Taiwan

4 National Taiwan University Hospital, Taipei, Taiwan

5 Sujan Cancer \& Surgical Hospital, Amravati, India

6 Mackay Memorial Hospital, Taipei, Taiwan

7 Division of Hematology-Oncology, Department of Internal Medicine, Taichung Veterans General Hospital, Taichung, Taiwan

8 Internal Medicine, College of Medicine, China Medical University, Taichung, Taiwan
9 Pyatigorsk Oncology Dispensary, Pyatigorsk, Russia

10 Ruby Hall Clinic, Pune, Maharashtra, India

11 Li Ka Shing Faculty of Medicine, The University of Hong Kong, Pok Fu Lam, Hong Kong

12 Boehringer Ingelheim Ltd, Bracknell, UK

13 Boehringer Ingelheim Canada Ltd, Burlington, ON, Canada

14 Boehringer Ingelheim Pharmaceuticals Inc, Ridgefield, CT, USA

15 Taipei Veterans General Hospital, Taipei, Taiwan 